\title{
Desafíos de la comunicación gubernamental en el cambio de época para las instituciones de Chimborazo, Ecuador
}

\section{Resumen}

\author{
Julio A. Bravo Mancero \\ Carrera de Comunicación Social
}

Universidad Nacional de Chimborazo, Riobamba, Ecuador

jbravo@unach.edu.ec

Estudio realizado para determinar si la comunicación gubernamental es asumida por las instituciones públicas de la provincia de Chimborazo, Ecuador, como un desafío para el cambio de época. Esta nueva arista de especialización reforzó la comunicación desde lo público sin ser un hecho reciente. La mirada de los profesionales hacia un estilo de comunicación más eficiente que vincule organización-personas obligó a tomar decisiones orientadas a que las acciones de gobierno lleguen a los diversos sectores como mecanismo de rendición de cuentas o de participación en la gestión. Como metodología se desarrolló un estudio de campo de tipo descriptivo durante dos momentos: un mapeo institucional y la aplicación de una encuesta. Los resultados indicaron que, si bien existe un fortalecimiento de la actividad, es necesaria una especialización de los comunicadores para la eficiente gestión de lo público.

Palabras clave: comunicación gubernamental, gobierno, gestión, toma de decisiones, Ecuador.

\section{The challenges of government communication in a new era for public institutions in Chimborazo, Ecuador}

\begin{abstract}
This study seeks to determine if public institutions in the Ecuadorian province of Chimborazo approach government communication as a challenge in changing times. Although not a recent development, this new field of specialization has strengthened public communication. Professional approaches towards a more efficient style of communication - one that links organizations and people - have led to more transparent government actions, enabling processes of accountability and participation. A descriptive field study was developed in two steps: through institutional mapping and a survey. Results indicated that, although communications have been strengthened, more specialized communicators are needed for a more efficient management of public affairs.
\end{abstract}

Keywords: government communication, government management, decision-making, Ecuador. 


\title{
Desafios da comunicação governamental na mudança de época para as instituições de Chimborazo, Equador
}

\begin{abstract}
Resumo
Estudo realizado para determinar se a comunicação governamental é assumida pelas instituições públicas da província de Chimborazo, Equador, como um desafio para a mudança de época. Esta nova arista de especialização reforçou a comunicação desde o público sem ser um fato recente. $\mathrm{O}$ olhar dos profissionais respeito de um estilo de comunicação mais eficiente que vincule organização-pessoas obrigou a tomar decisões orientadas a que as ações de governo cheguem aos diversos setores como mecanismo de rendição de contas ou de participação na gestão. Como metodologia se desenvolveu um estudo de campo de tipo descritivo durante dois momentos: um mapeamento institucional e a aplicação de uma pesquisa. Os resultados indicaram que, embora exista um fortalecimento da atividade, é preciso uma especialização dos comunicadores para a eficiente gestão do público.
\end{abstract}

Palavras chave: comunicação governamental, governo, gestão, toma de decisões, Equador.

\section{Introducción}

La comunicación gubernamental ha sido vinculada con las actividades del sector público, que, diversas como son, posibilitan el nexo con la comunidad. Esta perspectiva tiene el asidero teórico en autores como Bel Mallén (2005), Costa (2007), Elizalde \& Riorda (2013), Galindo (2011), Marí Sáez (2010), Massoni (2009), Pérez (2001) y Ulloa (2007). Es una parte esencial como apoyo de las áreas de decisión y las de acción. Es innegable que hoy las actividades de gestión tengan en la comunicación su soporte y ningún paso que se dé se aparte de ella. Esto obliga a una especialización ${ }^{1}$ de los comunicadores hacia un sector que los formará y abonará su experiencia sobre la base de todas las actividades de planificación, ejecución, acompañamiento y evaluación.

Es necesario aclarar que la comunicación no es más importante que la gestión, pero no existe gestión sin ella; todo hecho de carácter político decanta en uno comunicacional. Toda buena gestión tiene un equipo que se encarga de acompañar las acciones planificadas, que además deben evaluarse. Desde todo tipo de perspectivas, un gobierno debe motivar a su equipo a brindar acompañamiento para evitar tropiezos que incidan en el nivel de aceptación. La institución como un conglomerado de voluntades y circunstancias espera una adecuada proyección para alcanzar los objetivos. Los involucrados en el proceso deben asumir la misión y, sobre todo, desde lo comunicacional aceptar los abordajes para que la vinculación sea eficiente.

Vincular con la comunicación como eje significa delinear políticas ancladas con estrategias, actividades y recursos. Es ejercer una planificación coherente y coordina-

\footnotetext{
1 En Ecuador, la última década desarrolló la especialización en comunicación gubernamental con formalidad. Tiene asidero la situación política y organizativa de este país que ha experimentado cambios a todo nivel. Con rapidez encontró seguidores y detractores. Su esencia genera debate y desde la academia su análisis arroja resultados como la presente investigación que integró a profesionales en ejercicio, docentes y estudiantes.
} 
da, medible y evaluable. En esta etapa, los resultados dependerán del acompañamiento coherente.

Por esta razón, para investigar la realidad de la comunicación gubernamental en Chimborazo, provincia ubicada en el centro del Ecuador, a 273 kilómetros de Quito, se planteó el análisis de los desafíos de la comunicación gubernamental en el cambio de época para las instituciones de Chimborazo. Se evidenció de qué manera se realizan los procesos comunicacionales en la localidad, qué papel juega la planificación, qué se espera del vínculo institución-comunidad, cómo miran los ciudadanos la gestión institucional, cuáles son los perfiles de quienes dirigen el área, desde qué perspectiva se atiende las áreas centrales de decisión y las de acción, y cuáles son las oportunidades para los profesionales si deciden especializarse en este campo.

El estudio llevado a cabo centró su posición en el hecho de que en el área estudiada lo público despertó de la emergencia por ejercer una comunicación participativa como instrumento para construir el cambio, el involucramiento de actores y lo más coordinado posible ${ }^{2}$.

Para responder la interrogante se determinó como objeto de estudio de la comunicación gubernamental a los directores o responsables de comunicación durante dos momentos: un mapeo de instituciones y una encuesta; la información obtenida tiene la categoría de relevante y está contrastada con lo que se expone en la fundamentación teórica.

El análisis tuvo como punto de partida el objeto de estudio, es decir, qué es la comunicación gubernamental y cómo funciona la herramienta de vinculación de la institución con la comunidad; cuál es el proceso de la toma de decisiones y las acciones que demandan planificación, es decir, el empoderamiento de los involucrados. Evidenció la importancia de la estrategia para vincular lo institucional con la gente y la forma de hacerlo. Estableció la relevancia del mito de gobierno para posicionar la institución en los públicos, los componentes, cómo se la construye y la forma de comunicarlo.

El siguiente apartado del estudio describe la metodología empleada para realizar la exploración, la cual corresponde a una investigación de campo de tipo descriptiva. La población escogida fue de 33 directores departamentales de comunicación, delegaciones de ministerios, gobiernos autónomos descentralizados, entre otros; al ser un número manejable no se trabajó con muestra; se utilizó como técnica la observación y su instrumento fue la ficha. Para realizar la encuesta la herramienta fue el cuestionario.

\footnotetext{
2 En lo que cabe, porque a todo nivel existen procesos verticales que no pueden evitarse; orientados desde la dirección la jerarquía todavía pretende ordenar lo que debe atenderse, cuándo hacerlo, con qué recursos se cuenta, la hoja de ruta, los criterios de evaluación, la retroalimentación y la proximidad con la gente. En Chimborazo, como en las otras provincias ecuatorianas, persiste un fenómeno: la centralización de las actividades y ese esperar que todo se resuelva en un nivel jerárquico superior para poder caminar; incluidos los planes de comunicación que son una decisión que va desde lo macro a lo micro.
} 
Los instrumentos fueron aplicados por los estudiantes de la Universidad Nacional de Chimborazo (UNACH), Carrea de Comunicación Social, cátedra Relaciones Públicas, sexto semestre ${ }^{3}$.

Los resultados obtenidos demostraron que las instituciones de Chimborazo potencian la interacción con los usuarios a través de la utilización de las TIC, fundamentales en la sociedad de la información; si bien desde las instituciones se desarrollan actividades de comunicación gubernamental, la especialización de los profesionales todavía es una tarea pendiente; la planificación y el direccionamiento del mito de gobierno, la forma cómo se comunica deben tomar un giro que garantice eficiencia para la consecución de los objetivos. Desde la academia deben apuntalarse procesos de formación en posgrado.

\section{Desde lo comunicacional}

La comunicación es un hecho social (Rizo García, 2012), sinónimo de interacción, de significado, de retroalimentación y de afectos. Su dimensión tiene complejidad y su estudio, distintas aristas desde las que los teóricos elaboran sus propuestas. No ha alcanzado la categoría de ciencia [...] es un objeto de estudio. Tampoco es una disciplina, porque está en el camino de otras que intentan explicar fenómenos sociales-comunicacionales. Para conseguirlo y entenderse mejor, hay que considerar la comunicación entre humanos como una herramienta imprescindible. Tiene una dimensión de ciencia en construcción y su debate persiste en este siglo. Es un eje que asigna movimiento a todo. Un eje trans, inter y multidisciplinario sin discusión.

Es importante pensar que la investigación sobre los medios de difusión masiva y/o sobre las industrias culturales no es necesariamente investigar comunicación (Sánchez Ruíz, 2002). Su misión está más allá y por eso alcanzó la categoría de ciencia encrucijada. De igual forma, cada vez son menos las manifestaciones partidarias del carácter únicamente disciplinar de la comunicación (Silva \& San Eugenio, 2013). La comunicación es un cruce de múltiples caminos y, por lo mismo, no es reciente su estudio y profundización en Europa, Estados Unidos y América Latina.

Los esfuerzos apuntaron a la profundización como un imperativo de los aspectos comunicacionales. El mundo no es el mismo y en él, la comunicación —imprescindible como siempre- sigue siendo un eje. Lo global y lo glocal confluyen en una sucesión de actividades que entregan sentido y a partir de ellas se efectúan las construcciones.

\footnotetext{
3 Esta temática motivó que en octubre de 2014 se desarrollase un ciclo de conferencias organizado por la Carrera de Comunicación Social de la UNACH, con la participación del gobernador de Chimborazo, de alcaldes y de directores de comunicación. En el evento se debatieron los alcances de la comunicación gubernamental en la nueva época, el soporte y la nueva tendencia, la aplicabilidad de los procesos, las perspectivas y proyecciones, y la necesaria especialización.
} 
Aunque sugiera como una conclusión parcial, el fenómeno comunicacional no puede alejarse de lo cotidiano porque no es una repetición de actos sino el encuentro del significado desde la importancia de ¿qué hacemos y cuál es la intencionalidad?

La comunicación no puede apartarse de lo que se hace y lo que se piensa. Estar en construcción y en movimiento tiene desafíos y retos. Los comunicadores y las ciencias de apoyo intentan descubrir verdades que siempre quedaron a medias, tan vigentes después de décadas de propuestas. Ahí está la importancia de la comunicación gubernamental para vincular lo público con la comunidad.

\section{La nueva época}

A la nueva época - sociedad de la información - se la evidencia desde la década de los sesenta del siglo pasado, caracterizada por la necesidad del ser humano por coexistir en un tipo distinto de sociedad al que estaba acostumbrado. Privilegia el acceso a la información generada por otros y es un camino sin retorno. Edgar Tello sostiene que generó la brecha digital que visibilizó la diferencia de oportunidades de desarrollo para quienes tengan o no acceso a las tecnologías de la información y la comunicación. "Internet es bastante más que una plataforma tecnológica para el intercambio de información" (Tello, 2007, p. 2). Lo transforma todo y visibiliza la desigualdad social desde la perspectiva del investigador, no por el acceso a los recursos materiales sino a los patrones digitales. Un nuevo paradigma que asigna a la tecnología un rol causal en el ordenamiento social, ubicándola como motor de desarrollo económico.

En este proceso transformador están inmersas personas, colectivos e instituciones que se desenvuelven en un escenario - insospechado hasta hace poco- orientado por la inmediatez y la profundidad, que visibilizó el deseo del ser humano por encontrar mayor igualdad, solidaridad, libertad y equidad.

La sociedad de la información, que se centra en la persona -integradora y orientada al desarrollo-, distingue dos factores: lo externo se encuentra en el entorno: la economía, la legislación, la cultura, la formación, la promoción o las actitudes. Y lo interno, que se desdobla en tres ejes: los usuarios (ciudadanos, las empresas-asociaciones y las administraciones públicas); las infraestructuras (los servidores, las redes y los terminales); y los contenidos (los servicios y la infomediación con contenidos tangibles e intangibles).

¿Qué afectación tiene lo institucional? La vida de la sociedad está caracterizada por un modelo social y virtual; hay que estar en la internet para trascender y desde allí surge la nueva definición de familia, trabajo y empresa. El conocimiento y la información son elementos decisivos en los modos de desarrollo. La adaptación a los no lugares trae otros valores, saberes y gustos, con lo multimedial como esencia de lo atemporal y lo a-espacial, aspectos decisorios para una eficiente labor de la comuni- 
cación gubernamental; lo físico ha sido reemplazado por lo virtual con mejores posibilidades para la eficiencia de acciones conjuntas y no individuales, bi y no unidireccionales. Los contenidos acercan al ciudadano, lo aproximan, lo hacen interactuar y tomar decisiones. Los accesos al usuario inciden en su conducta e influyen en su mundo. La información produce otro tipo de relación individual, social e institucional; la tecnología es un factor clave para lo cotidiano; las TIC están transformándolo todo y han individualizado-personalizado las acciones; lo digital rompió el esquema y en los no-lugares se escenifican espacios de interacción que garantizan formas distintas de construcción de sentido.

Rosa María Torres (2005) sostiene que las transformaciones que acompañan la acelerada introducción en la sociedad de la inteligencia artificial y las nuevas tecnologías de la información y la comunicación son profundas.

Para Tello:

El concepto de sociedad de la información hace referencia a un paradigma que está produciendo profundos cambios en nuestro mundo [...]. Esta transformación está impulsada principalmente por los nuevos medios disponibles para crear y divulgar información mediante tecnologías digitales. Los flujos de información, las comunicaciones y los mecanismos de coordinación se están digitalizando en muchos sectores de la sociedad, proceso que se traduce en la aparición progresiva de nuevas formas de organización social y productiva $(2007$, p. 3$)$.

Desde esta aseveración, Ecuador no está exento de esta forma de interacción. En la última década la inversión gubernamental y la privada iniciaron la construcción de una nueva manera de desarrollo basada en los patrones digitales que incide en la forma como se hace comunicación gubernamental. En este contexto, las actividades de las organizaciones miran hacia un conjunto de acciones interrelacionadas, el servicio se fortaleció como tal y los públicos alcanzaron la categoría de usuarios, con diferencias marcadas sobre la base de la fundamentación teórica y la praxis. La estructura organizacional redefinió roles y estatus; lo horizontal tiene mayor relevancia y los vínculos se construyen a partir de los trazos de las hojas de ruta de la nueva teoría estratégica, la que se precisará en los siguientes párrafos.

\section{¿Hacia dónde va la comunicación gubernamental?}

Se define el territorio gubernamental como el espacio de acción sistémica y discursiva de un organismo en posición de coordinación social, que controla recursos públicos sobre la base de sus definiciones institucionales. Las posibilidades de la acción política desplegadas desde un territorio están determinadas por recursos normativos, estatutarios, humanos, de acción, materiales, informacionales y relacionales, asegura Irving Berlín (2007). 
Los gobiernos locales tienen retos generales, como niveles de gobierno que sostienen relaciones intergubernamentales y particulares, la elaboración de políticas públicas para el desarrollo de zonas específicas del espacio público, entendido no ya como un territorio simbólico sino como espacio real donde la circulación y la socialización de las personas se hace posible. En el campo de la comunicación se construye el diseño de estrategias mediante acciones y discursos que impacten en la vida cotidiana de los ciudadanos y de las organizaciones institucionales que les sirven de entorno.

Berlín afirma:

La comunicación gubernamental en los espacios locales reclama una definición operativa que no contradiga los fundamentos de la comunicación política y esté conforme con las características de los territorios gubernamentales y sus márgenes de decisión. Se define aquí comunicación gubernamental al conjunto de procesos mediante los cuales un organismo territorial de poder público selecciona -incluyendo y excluyendo- las decisiones de gobierno para intervenir en zonas de consenso o de conflicto de tal manera que coordine eficazmente la transformación social y la participación ciudadana mediante el diseño de estrategias y tácticas de acción política y discursiva (2007, p. 97).

En este plano, primero surge la estrategia para influir, afectar o activar a las personas. Ahí está la clave y la importancia de este acto. La comunicación debe entenderse como teoría, doctrina o práctica. Nada funciona ni tiene efecto sobre los seres humanos, ni siquiera la estrategia que se considere de avanzada si no se la vincula con la comunicación. "Esto ha sido algo más permanente que cambiante. La estrategia social, la que necesita afectar a las personas, no puede prescindir de la comunicación" (Elizalde \& Riorda, 2013, p. 9). Si lo cotidiano de la vida gira en torno de la comunicación, las actividades públicas no pueden desvincularse. Si lo hicieran las organizaciones quedarían invisibilizadas y no trascenderían.

A partir de seis ejes, el acto de comunicación gubernamental delimita los desafíos y determina escenarios de desenvolvimiento para garantizar efectos de corto, mediano y largo plazo. Mejorar para perdurar, desde el componente político eleccionario; reelegirse es bueno cuando se dijo algo nuevo cada día; es como una semilla que entregará frutos al final de una gestión que puede posicionarse como el principio de otra.

Comunicación directa, actuación con partners o socios, mitos de gobierno, criterios de organización ágiles para el cambio, convergencia como paradigma, innovación y tecnología, microtargeting y comunicación como legitimación, son los elementos con los que se trabaja con la intención de cimentar lo gubernamental.

La vinculación de lo institucional con la gente demanda una eficiente toma de decisiones que solo es posible cuando el director de comunicación (dircom $\left.{ }^{4}\right)$ ha desarro-

\footnotetext{
4 El dircom es un organizador del trabajo; su liderazgo hace que la toma de decisiones sea más sencilla, desde la aproximación que deja la formación (fundamentación teórica) y la praxis (experiencia), dos hechos inseparables que decantarán en una gestión con reconocimiento por la consecución de los objetivos estratégicos. Y eso redunda en el desarrollo institucional.
} 
llado habilidades imprescindibles para ejercer su actividad. El eficiente liderazgo desde una dirección de comunicación mejorará la capacidad de recepción y la respuesta de la gente como fin último de todas las decisiones que se tomen, máxime del involucramiento en una actividad reciente que volvió incluyente la gestión.

Rizo García ${ }^{5}$, desde los imaginarios sobre comunicación, elabora su propuesta y la enmarca en la categoría de hecho social que, aplicado a lo organizacional, redefine desde lo individual hasta lo colectivo y desde lo simple hacia lo complejo; es sinónimo de interacción cuando se entiende que la gestión pública no cumple sus objetivos si no se refleja en lo que las otras personas esperan que se haga y está claro que la soledad no es un componente en la gestión, ya que ella no posibilita retroalimentación ${ }^{6}$; de significado, en la dimensión en que cada uno de los mensajes deben aproximar a la cotidianidad de los mandantes, lo que en el día a día se hace; y de afectos, en la medida en la que los funcionarios se acerquen las personas y entreguen su espaldarazo a las actividades por mínimas que sean.

La comunicación directa rechaza de manera definitiva la mediación de la prensa como única forma de difusión y encuentra en la proximidad a los públicos como el mecanismo útil para cumplir los objetivos. La actuación con partners o socios es el mecanismo adoptado por las instituciones para no aislarse del mundo y ahí está la correlación que Rizo García propone. Se trata entonces de actuar con otros actores políticos pese a la rivalidad que finalmente es subjetiva cuando empieza la función. "Gestionar en red es el nuevo paradigma de la gestión" (Elizalde \& Riorda, 2013, p. 10).

Definir la herramienta simbólica que le entregue sentido a la construcción social y política; que evidencie una carga ideológica capaz de generar consensos y alejar los disensos es la asignatura que le corresponde a la elaboración de mitos de gobierno. Los criterios de organización deben ser ágiles y flexibles ya que la comunicación presenta el rostro visible de los cambios. El personal que intervenga en la actividad necesita formación y preparación para garantizar la transformación. El escenario en que el mundo se desenvuelve cambió hace dos décadas. Se hace necesaria la intermediación y la urgencia por comprender que las audiencias son migrantes. Entonces, convergencia no es sinónimo de eficacia sino de una acuciante necesidad. Organizar la innovación debe entenderse en un amplio sentido. El microtargeting individualiza segmentos, crea bases de datos específicos para esta tarea y a partir de la comunicación como legitimación hay que entender que la clave está en la comunicación antes que en el hecho.

Ningún acto de comunicación se aparta de la política y es la planificación estratégica de la comunicación gubernamental la que establece las líneas gruesas. Deben acep-

\footnotetext{
5 Referenciada por el autor en la delimitación de la comunicación como eje trans, inter y multidisciplinario, por ser una apreciación contemporánea y de contenido académico.

6 La interacción garantiza la perdurabilidad de la comunicación gubernamental y entrega un lugar a cada uno de los actores que en ella se desempeñan.
} 
tarse transformaciones mutuas y encararlas desde el componente teórico-práctico. La política es una interacción constante de símbolos, un proceso articulable y predecible en el espacio de los mensajes emitidos y recibidos. Vale recordar que política se hace con comunicación.

Lo político es un hecho comunicable; siempre que haya política habrá comunicación. Desde la decisión política se hacen los análisis de variables comunicativas que evidencian ganar o perder reputación, credibilidad y legitimidad. Siempre habrá adaptabilidad política a las decisiones comunicacionales (Graber citado por Elizalde \& Riorda, 2013).

La clave para una buena comunicación política está en la interacción con la gente, aprovechando los nuevos medios y no uno en especial (modelo analógico). “Comunicar una decisión, una acción, es más fuerte que la acción misma.Y decimos -fuerte- en el sentido del término", Joan Costa (1999, p. 128). Insiste que a partir de esas ideas cualquier decisión, cualquier actuación que se emprenda, además de ser realizada tiene que ser comunicada. Y después evaluada en sus efectos. Es lo que se llama comunicación por objetivos.

\section{Decisiones como proceso estratégico}

La contemporaneidad trajo la nueva teoría estratégica (NTE), propuesta delineada por el profesor español Rafael Alberto Pérez (2001) que provocó siete cambios: el primero se da en el paradigma central, como un prototipo integrador de la comunicación y lo complejo sin lugar para la fragmentación; su misión reemplaza los viejos por nuevos mapas mentales que transforman la realidad físico-social condicionada por las visiones del mundo proporcionadas por las teorías, modelos y paradigmas que tienen su génesis en la educación formal y no formal recibida. La sociedad reclama un giro $\mathrm{y}$ otros roles que dejen de lado las estrategias de forma dual, fragmentaria, unidimensional, estática, causal y lineal, ligada a lo cuantitativo. El segundo está anclado en el sujeto; encasilla al hombre como un individuo relacional; las estrategias y las decisiones son tomadas por el individuo pero que, para lograr sus metas, necesita de otros. Todo tipo de aislamiento lo deja fuera. Recuperar lo humano le preocupa a la NTE. El tercero es la organización; el hombre por sí solo no puede alcanzar sus metas, necesita asociarse y pasar de lo individual a lo colectivo. Esta unión lo obliga a definir objetivos y metas de corto, mediano y largo plazo; la cooperación de todos es la base para la acción. ¿Y qué ha posibilitado? El surgimiento de otra concepción de organización: sistémica, coevolucionista, innovadora, conectiva, significativa y socialmente responsable. No se trata de una organización ideal, sino de una posible. El cuarto cambio, el enfoque, es pasar del conflicto a la articulación para perdurar. La NTE busca pasar de un enfoque conflictivo a otro más comprensivo y dialogante. El nuevo enfoque teórico 
revela conductas articuladoras, en las que escuchar, armonizar y reconducir a un cauce común se presentan como claves. Esta perspectiva señala el camino: reemplazo de la actuación singular por la plural. El quinto: la matriz de estudio; pasar de la economía a la comunicación y en la praxis la estrategia se convierte en un campo de conocimiento que articula las cuatro anteriores y que se adapta a las exigencias que se encuentran en los entornos. Aquí la comunicación se convierte en un corpus teórico que ofrece una matriz social, relacional, sin más limitaciones que las humanas. Sirve para describir relaciones fácticas y aquellas posibles, imaginadas, las que se alojan en el pensamiento y en el deseo. No solo las interacciones simbólicas sino también otros espacios de hibridación y encuentro.

Los cambios sexto y séptimo se abordan desde el método y la metodología. Por el contrario de los paradigmas tradicionales sobre la base de la fragmentación, la NTE busca un mundo fluido, complejo, a veces caótico, multidimensional y en red.

Pensar desde la estrategia es hacerlo desde el lugar de los otros; es anticipar las reacciones y cambios de decisión, de emoción y de acción; la fundamentación teórica cumple su rol y le entrega - al director de comunicación- el estatus de estratega si aprovecha la oportunidad de la actividad. La autoridad y la asesoría de la comunicación gubernamental deben convencerse de que el proceso de intelección tendrá como efecto la premisa: no actúe solo porque no lo está.

Jesús Galindo (2011) refirma que la comunicación estratégica se desenvuelve en medio de la emergencia. En aquella toman partido varios jugadores y no uno específico; las acciones desde lo institucional son de doble vía. Comparte objetivos comunes y para alcanzarlos debe definirse la audiencia, los contenidos de los mensajes y los medios que se utilizarán para difundirlos. Su elección está sujeta a la ejecución, al control y a la evaluación, tres etapas presentes en todo acto comunicativo con la gente como eje sobre el cual gira todo.

La figura de pensamiento estratégico sigue ahí, pero con la mediación de la comunicación desde la interacción de los jugadores. Mientras haya más involucrados los resultados serán mejores. Es decir que la diversificación y el incremento de participantes garantiza el éxito. Está claro que la comunicación gubernamental motivó el giro hacia la comunicación conflicto (consenso en detrimento de la información). La clave está en multiplicar los participantes y en tender puentes para compartir no solo experiencias sino actitudes que contribuyan el cambio y mejorar los criterios de ciudadanía.

De nada sirven los pasos por caminar si no se mira el entorno; en él están los insumos para mejorar, avanzar y proyectarse al futuro. El equipo de trabajo tiene que comprender que la estrategia que se utilice está condicionada por la presencia, las percepciones y las decisiones de otros jugadores cuya acción es importante como agente regulador de actividades, significados, sentido y construcción de ciudadanía. De la 
misma manera, está en correlación con las orientaciones de la sicología y con el análisis sociológico.

Lo externo desde el hecho comunicacional es como un destino anticipador de conformación de redes; los integrantes de la organización tienen por misión desarrollar relaciones con actores - considerados claves - y todos ellos están en el entorno institucional. "La escucha externa (a veces con retroalimentación) en consecuencia, comunicación para mantenerla y estimularla" (Bartoli, 1992, p. 101).

José Ignacio Bel Mallén dice que hoy la comunicación externa en las organizaciones dejó de ser "una moda pasajera, limitada a obtener una presencia en los medios de comunicación" (2005, p. 158). Al mismo tiempo explica (Bel Mallén, 2005) que en las organizaciones, sin importar su tamaño, la comunicación es un eje fundamental; todos los sistemas de comunicación externos están implícitos en cada una; a mayor proyección pública, mayor comunicación externa para posibilitar la identificación y el posicionamiento; las relaciones organización-público se canalizan de una forma o de otra a través de medios o sistemas de comunicación externa; los responsables ejecutivos de las organizaciones deben conocer de comunicación e información - a mayor responsabilidad en una organización mayor conocimiento del manejo comunicacional-; todo director de comunicación debe convencerse de que no son sencillas las relaciones con los medios ni con otros profesionales en el manejo y dosificación de la información, las dos partes tienen un mismo interés: institución y ciudadanía. Hoy los medios no son el único mecanismo para llegar al público: la organización vive en el centro del público, de los medios y sus clientes; por eso necesita cuidar los mensajes que emite.

José Barroso Gómez (2013) considera que para efectos de las estrategias de comunicación es vital comprender las relaciones humanas como interacciones simbólicas en las cuales el diálogo posibilita la intercomprensión, a la manera de juegos de suma distinta de cero: mediante el diálogo es posible que los jugadores encuentren objetivos comunes y puedan lograr beneficios que serían imposibles de lograr mediante la mera confrontación entre jugadores-luchadores.

\section{Del mito de gobierno a la elaboración de mensajes}

Existen dos caminos en la gestión de la comunicación gubernamental que aclaran el panorama antes de la toma de decisiones. Por una parte, se reconoce que los gobiernos requieren de una buena aceptación y calificación; y por otra, saber perdurar en el ánimo ciudadano ${ }^{7}$. Los funcionarios que desarrollan una gestión piensan que el período en curso debería convertirse en la antesala de uno nuevo.

\footnotetext{
El ánimo ciudadano es una categoría subjetiva.
} 
Se define al mito de gobierno como:

Un sistema de creencias, coherente y completo, que da sentido y orientación al gobierno y que ofrece la dirección estratégica y expresa el sentido último de la gubernamental (Elizalde \& Riorda, 2013, p. 27).

Para estos autores, el mito de gobierno debe crear pertinencia y pertenencia a los involucrados de la organización. Va más allá de la misión y de la visión, de soñar con una organización con vocación de servicio, sino que se acerque a la comunidad, que la haga partícipe de todos los procesos y la mejor manera de hacerlo es aproximarlo a las decisiones que incidirán con sentido colectivo.

La comunicación del mito de gobierno encauza las actividades internas (identidad) y las externas (imagen). Desde la reflexión es necesario un tercer nivel para operativizar ese vínculo interior-exterior: y la comunicación corporativa, que permite transformar identidad e imagen para evitar el menor ruido posible en los públicos.

$\mathrm{Si}$ se pone énfasis en que las actividades internas se vinculan con la identidad, la perspectiva de Costa (2007) tiene coherencia; su propuesta establece como componentes: identidad cultural, identidad verbal, identidad visual, identidad objetual, identidad comunicacional. Las cinco categorías definirán lo que la institución es, para qué sirve lo que hace, su estilo y conducta, y las relaciones que establece con el flujo de información. Esto debe quedar claro para el comunicador.

Sandra Massoni (2009) puntualiza que el comunicador es un profesional con capacidad de interpelar la dinámica social para operar crítica y valorativamente en su dimensión comunicativa. Su tarea, al reconocer la diversidad sociocultural, es indagar los posibles puntos de articulación de las diferencias en función de los intereses y las necesidades de grupos sociales que se reconocen como bien distintos, para operar desde allí en relación con un objetivo de transformación cognitiva macrosocial.

Vincular gobierno y ciudadano es hacerle sentir que es parte de él, clave para el logro del mito. Toda construcción de mensajes pasa por la definición del mito de gobierno y por su anclaje como base de la arquitectura de la comunicación ${ }^{8}$. Desde la propuesta de César Ulloa Tapia (2007, p. 70) es imprescindible "identificar las formas de comunicación que se comparten antes de imponer expresiones”.

Si la intención es modificar la conducta de quienes están relacionados con las acciones, existen mensajes: que enfocan (tienen como referencia a otros mensajes, propios o ajenos llamados metamensajes); que dan versiones (son historias que narran de una perspectiva particular para persuadir o influir); que son como actos (definen de modo público - manifiesto- y compartido - social - las intenciones que se man-

\footnotetext{
8 Así llama el autor a todo lo referente a las etapas de diagnóstico, direccionamiento estratégico, portafolio de intervención, control y monitoreo, evaluación que son piezas del plan de comunicación. Empezarán desde la línea base que es la política de comunicación, definición de estrategias y establecimiento de acciones, sobre la certeza presupuestaria y la decisión política. Esta última, vital para el desarrollo de una gestión.
} 
tienen en las acciones; que señalan (generan una reacción, son de tipo impersonal y sistémico, dirigidos a todos).

Desde esta perspectiva, la mitad de los directores de comunicación de las instituciones de la provincia de Chimborazo conocen el fundamento del mito de gobierno, lo que facilitó el presente estudio; un logro para el manejo de la comunicación gubernamental como área especialización.

\section{Comunicar para gobernar}

Los gobiernos deben apartar la forma jerárquica y multilateral en su actuación; la legitimación y el consenso son como la piedra angular de todo. Si no se comunica no habrá trascendencia y si no hay trascendencia los objetivos no se alcanzan. "La comunicación ya no es para los gobiernos un ejercicio cotidiano, representa el vehículo indispensable, se puede y se debe transmitir su esencia" (Elizalde \& Riorda, 2013, p. 47).

En el marco de la contemporaneidad no es suficiente — según Javier Sánchez (2013) - con ser honesto, distribuir el presupuesto en áreas sociales, manejar bien la retórica del discurso, para ser valorado como un buen gobierno. El nuevo contexto obliga a efectuar el diagnóstico de las necesidades de la gente, que es básico para la toma de decisiones. Para cumplirlo se debe: conocer la demanda ciudadana, definir el concepto de gobierno, establecer los temas de coyuntura, conformar estrategias adecuadas para conducir la agenda pública, homogeneizar los mensajes oficiales y diseñar una estructura organizacional adecuada para los esfuerzos de comunicación social.

Un eficiente ejercicio de la comunicación debe incluir: políticas de comunicación social, estructuras operativas y un plan de trabajo. El modelo de comunicación permitirá la integración: estrategia-ejecución de las acciones comunicativas; hay que distinguir entre comunicar e informar.

Comunicar bien es la resultante de la profunda reflexión que le permita decidir al gobernante sobre situaciones que pueden ser tensas, arriesgadas y que a veces debe hacer sin tiempo ni asesoría oportuna. "Las organizaciones son microsociedades establecidas por la relación entre personas" (Véliz Montero, 2014, p. 33) y requieren de canales, mensajes, datos e intenciones para cumplir propósitos.

Lo gubernamental anclado en la comunicación denota la producción e intercambio de sentidos, interpretación y creación de nuevas expresiones. Como fenómeno gestor de interacción sociocultural incide en su producción, calidad, procesos y resultados. La elaboración del discurso en los mensajes que se pondrán a disposición ciudadana debe ser una actividad reflexionada que entreteja un profundo sentido de persuasión.

Para Edilia Escudero: 
La comunicación en la organización es fundamental porque es la encargada de conectar, vincular, unir y enlazar los procesos, las actividades, la trama de relaciones e interacciones, así como los grupos de interés internos y externos de los significados y símbolos que se deciden negociar y tranzar para fortalecer la identidad en el microambiente y proyectar la imagen en el macro ambiente (2014, p. 168).

La vida de la organización no puede apartarse de la comunicación; ella es su esencia y de su planificación fluyen las actividades. Es por esta razón que su dimensión estratégica la posiciona como un eje que establece vínculos, logra consensos y transforma lo social.

Sin embargo, Pérez plantea que la organización cambió el viejo paradigma piramidal y jerárquico por uno sistémico, conectivo y significativo. Lo comunicacional no enfoca escenarios de persuasión sino de dirección'. Se trata de conectar las inteligencias presentes en la organización e interconectar los aportes de cada componente para motivar sinergias que contribuyan a la consecución de objetivos comunes:

La comunicación pasa así a ocupar un papel constituyente y no meramente instrumental. De ahí el énfasis que autores como Levi-Strauss y Castoriadis, Maturana y Varela, Luhmann y Greenfied han puesto en la importancia de la conversación y la construcción social de los significados (Pérez, 2012, p. 153).

\section{Trabajar en una campaña permanente}

Afrontar una campaña es una suma de esfuerzos por un tiempo determinado con el equipo de trabajo, la utilización de herramientas y un presupuesto limitado para llegar al poder. Pero ¿qué ocurre cuando llega al cargo? ¿qué se hace para mantener la adhesión del electorado? ¿cómo sumar a los que no votaron por él? ¿cómo mantener la confianza y cómo incrementarla durante el mandato? ¿habrá una siguiente postulación?

Por ejemplo, en las elecciones del 23 de febrero de $2014^{10}$, Carlos Aguirre, actual alcalde de Chunchi, cantón ubicado en el sur de la provincia de Chimborazo, en una encuesta realizada treinta días antes de la elección, contaba con una intención del voto del 46.2 por ciento y ganó en los comicios con el 43.10 por ciento. Para que esta realidad guardara coherencia con lo expresado, el funcionario debería reforzar el trabajo para persuadir al 56.90 por ciento de los electores que no habían votado por él; adherirlos al proyecto político y proyectar su gestión sobre la base de los sufragios obtenidos. Y el único camino era mantenerse en campaña permanente: utilizar el cargo para construir una amplia y suficiente base de apoyo popular.

De este modo, existe la evidencia de que los ciudadanos se volvieron cada vez más independientes políticamente, sin fidelidad partidista; la campaña permanente ayuda a mantener a la gente conforme y de su lado. La promesa de campaña electoral debe tra-

\footnotetext{
9 La tarea directiva consistirá en saber establecer qué sentido y qué significación ha de generar una organización.

10 El equipo de investigación de la Carrera de Comunicación trabajó en la medición en el cantón Chunchi.
} 
ducirse en la campaña gubernamental permanente; es decir, el mensaje debe responder $\mathrm{y}$ atender las necesidades y expectativas básicas del electorado.

Blumenthal (citado por Elizalde \& Riorda, 2013) identifica cuatro características de la campaña permanente: 1) los políticos desean mantenerse y acrecentar su imagen pública; 2) la sistematización de la información es un recurso que evidencia la aceptación o rechazo a una gestión; 3) la campaña permanente es una práctica recurrente por la modificación de la reglamentación electoral en todo el mundo; 4) gobernar en campaña es el instrumento para sostener la popularidad. Para cumplirlas se recurre a consultores políticos (comunicadores sociales), profesionales de la comunicación con experticia en la realización de diagnósticos o un equipo de comunicadores para apuntalar la planificación, la ejecución y la evaluación.

\section{¿Construir la imagen pública?}

Conseguir que un gobernante entre en la mente de un ciudadano y no se aparte de ella merece las siguientes precisiones. En campaña el candidato fue objeto de señalamientos y de ataques que prolongan su vida hasta la gestión. Al usar una estrategia eficiente de comunicación la borrará, aclarará o desvirtuará; hay que lograr que los ciudadanos crean que el gobernante está pendiente de ellos durante el mandato y no de lo que dijeron de él durante el proceso previo a su elección.

Una gestión gubernamental tiene que definir desde el comienzo los objetivos para construir la imagen pública del gobernante y trabajar para que esta se posicione en la mente del ciudadano. Si no se logra identificar bajo qué características particulares el gobernante construirá su imagen pública y la de su gobierno, existirá un incendio por apagar que impedirá sostener un mandato. El inicio de una gestión llega acompañado por una expectativa alta en el cambio de gobierno, traerá escepticismo respecto de las propuestas de campaña, sobre todo si no existió el cumplimiento de quienes dejan el cargo.

Surge, entonces, la construcción de la identidad simbólica manifestada en la cimentación de imaginarios desde los cuales los ciudadanos se interrelacionen, interactúen, lleguen a consensos y disensos, tomen decisiones y construyan sentido. Si la comunicación política tiene como objetivo generar consensos, el director de comunicación se encargará de tender los puentes para conseguirlo.

El mecanismo para alcanzar una buena imagen pública es el diálogo, "proceso con el cual las personas suspenden los cambios defensivos con el objeto de permitir el libre flujo de la exploración de las figuraciones y creencias propias y de los demás", precisa Mónica Valle (2005, p. 151). La comunicación como herramienta abre posibilidades y marca el camino hacia el desarrollo como actividad compartida. 


\section{Metodología}

La presente es una investigación de campo y se desarrolló durante dos momentos: el primero, un mapeo de instituciones realizado en octubre de 2014; y el segundo, una encuesta dirigida a los directores de comunicación durante diciembre de 2015 y enero de 2016. Se trabajó con una población de 33 responsables de comunicacional; al ser manejable la población no fue necesario el cálculo de la muestra.

Para el mapeo institucional se identificaron los datos generales de la institución y si contaba con una dependencia encargada del manejo de la comunicación; el perfil del responsable, si su trabajo respondía a un plan y los recursos con los que disponía. Entre tanto, la encuesta tuvo un cuestionario de diez preguntas sobre comunicación gubernamental con énfasis en los sistemas que manejaban, las ventajas de su utilización, el mito de gobierno y sus componentes, y la adecuada toma de decisiones para sostener a un gobierno. Los datos obtenidos se agruparon en tablas, acompañadas por el análisis y la interpretación de los resultados.

También fue una investigación bibliográfico-documental. La fundamentación teórica expuesta como soporte para la realización del estudio se tomó de trabajos especializados en comunicación, comunicación: corporativa, gubernamental y organizacional, identidad e imagen corporativa.

\section{Discusión de resultados}

Los dos momentos de investigación citados en los aspectos metodológicos identificaron, en efecto, que la nueva época no es ajena a las instituciones de Chimborazo; se evidencian necesidades de coexistencia con la organización y la toma de decisiones. Es una realidad presente en la planificación de las actividades de comunicación.

Las tecnologías de la información y la comunicación abrieron la posibilidad para la inmediatez y también para la interacción, el fortalecimiento del vínculo y pasar de lo singular a lo plural en las actuaciones; la sociedad de la información pretende eso: un mayor acceso ciudadano a los servicios de la institución y la participación efectiva. Es democratizar a los espacios asignados para las minorías y entregar equidad en las oportunidades para los ciudadanos.

A continuación, se presentan los resultados obtenidos durante la investigación:

\section{Observación}

El estudio evidenció que existe preocupación en las autoridades de las instituciones porque la gestión de la comunicación se realice desde un área específica, cargo 
que no en todos los casos está en manos de un comunicador social ${ }^{11}$. El desempeño de quienes dirigen la dependencia se encuentra con una barrera por no contar con las herramientas necesarias; el equipamiento es fundamental para atender todos los requerimientos y cumplir con eficiencias las actividades asignadas. La planificación de la comunicación es un ejercicio desde el cual se establecen las prioridades institucionales en función de lo colectivo y asegurando la participación ciudadana en la toma de decisiones, garantizada en la Constitución Política del Ecuador.

La realidad institucional sobre el manejo de la comunicación desde un área específica evidencia una proximidad entre las entidades que sí lo poseen y las que no o están en proceso de consolidación.

El mapeo también reflejó la persistencia de espacios de comunicación atadas a departamentos de talento humano, vinculación con la colectividad, secretaría general, secretaría jurídica, relaciones nacionales e internacionales. El tratamiento de lo comunicacional requiere especificidad y autonomía para la toma de decisiones que redunden en la consecución de los objetivos (tabla 1).

Tabla 1. Mapeo de instituciones de Chimborazo.

\begin{tabular}{|c|c|c|c|c|c|c|c|c|c|c|c|}
\hline Categorías & & in & $\stackrel{0}{z}$ & 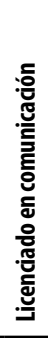 & 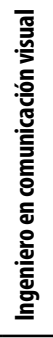 & 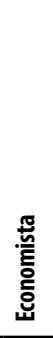 & 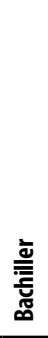 & 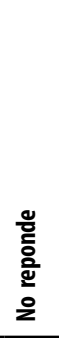 & 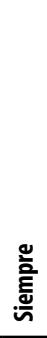 & 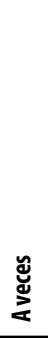 & 巳 \\
\hline \multirow{4}{*}{ Manejo de la comunicación } & Área específica & 58 & 42 & & & & & & & & \\
\hline & Perfil profesional & & & 48 & 12 & 3 & 9 & 27 & & & \\
\hline & Herramientas para la gestión & 36 & 64 & & & & & & & & \\
\hline & Planifica la comunicación & & & & & & & & 73 & 18 & 9 \\
\hline
\end{tabular}

Elaboración: Julio Bravo Mancero. Los valores que se expresan en la tabla son porcentuales

\section{Encuesta a directores de comunicación}

La aplicación del instrumento comprobó que la comunicación gubernamental es una parte fundamental para la mejora de la gestión con énfasis en el territorio guber-

\footnotetext{
11 Persiste el criterio de que la comunicación de la institución tiene que estar atada a otra dependencia o que la persona a cargo del área no debe ser un profesional del área. Al tener el puesto un cargo de libre remoción, por ser director departamental se lo escoge por afinidad sin que prime el criterio técnico al respecto. Los procesos comunicacionales quedan inconclusos cuando concluye el período para el que fue elegido o cuando el funcionario fue removido.
} 
namental -incluyendo y excluyendo- a la gente en las decisiones de intervención en procura de la transformación social (desarrollo); prevalece el sistema de comunicación horizontal frente a la vertical - nueva teoría estratégica-, lo que provoca un desplazamiento de la tradición jerárquica empleada para orientar las actividades desde la autoridad. Lo horizontal es una premisa del cambio de época. A partir de ella se establece la agenda, que vinculará la gestión de la organización con la gente. Es vital para el conocimiento y la atención de las necesidades de la gente, como aporte para la toma de decisiones (tabla 2).

Tabla 2. Utilización de la comunicación gubernamental.

\begin{tabular}{|c|c|c|c|c|c|c|c|c|c|c|c|c|c|c|c|c|c|}
\hline Categoría & in & 울 & $\begin{array}{l}\text { 퐁 } \\
\text { Nㅗㅎ } \\
\text { 오 }\end{array}$ & స్ & 을 & 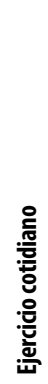 & 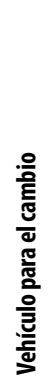 & 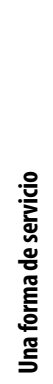 & 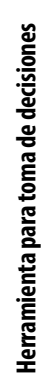 & 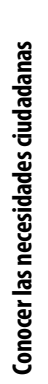 & 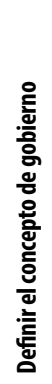 & 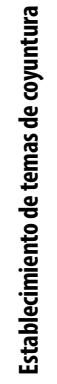 & 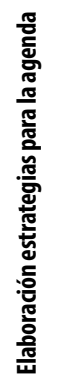 & $\stackrel{\check{\pi}}{\circ}$ & 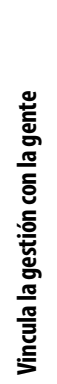 & 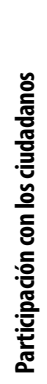 & 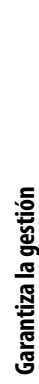 \\
\hline $\begin{array}{l}\text { La comunicación gubernamental } \\
\text { mejora la gestión }\end{array}$ & 87 & 13 & & & & & & & & & & & & & & & \\
\hline Su sistema de comunicación es & & & 54 & 34 & 12 & & & & & & & & & & & & \\
\hline $\begin{array}{l}\text { La comunicación gubernamental } \\
\text { para su institución es }\end{array}$ & & & & & & 16 & 22 & 22 & 40 & & & & & & & & \\
\hline $\begin{array}{l}\text { La comunicación gubernamental } \\
\text { le permite a su institución }\end{array}$ & & & & & & & & & & 18 & 6 & 12 & 24 & 40 & & & \\
\hline $\begin{array}{l}\text { Desde lo comunicacional, } \\
\text { un gobierno }\end{array}$ & & & & & & & & & & & & & & & 47 & 25 & 28 \\
\hline
\end{tabular}

Elaboración: Julio Bravo Mancero. Los valores que se expresan en la tabla son porcentuales

Existe equilibrio en el conocimiento de lo que significa para la institución el mito de gobierno y que la identidad es el componente fundamental frente a la imagen de la organización. La forma en la que se comunica merece un giro que imponga el ritmo y socialice lo que significa para el colectivo. Será garantizar el involucramiento de los ciudadanos en las decisiones. Mantener el concepto de que mito es solo misión y visión - cuando en realidad se trata de: las creencias, los principios, los valores y la culturaplantea repensar la comunicación desde la gente, aplicar el pensamiento estratégico para multiplicar los participantes, hallar consensos y fortalecer la información para ampliar las perspectivas de participación. La nueva teoría estratégica busca que el viejo paradigma se modifique por uno nuevo que fortalezca la mancomunidad. Se reafirmó 
que el proyecto general de gobierno tiene como piedra angular la comunicación para el empoderamiento de la gente y que se involucren en las acciones. Se habla de incidir en la vida cotidiana de los ciudadanos y de las organizaciones, con enfoque en la nueva época y que lo comunicacional desplace los esquemas tradicionales (tabla 3 ).

El plan de comunicación define las actividades a corto, mediano y largo plazo como un documento que, partiendo del diagnóstico, delínea el direccionamiento estratégico, el portafolio de intervención, ejerce el control y monitoreo del proceso, y concluye con la evaluación.

Tabla 3. Definición del mito de gobierno.

\begin{tabular}{|c|c|c|c|c|c|c|c|c|c|c|c|c|c|c|c|}
\hline Categoría & in & 울 & 急 & $\begin{array}{l}\text { 뮨 } \\
\text { 늡 } \\
\text { 흔 }\end{array}$ & $\begin{array}{l}\text { 胥 } \\
\text { 竞 }\end{array}$ & 올 & 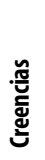 & :흘 & :흔 & $\frac{\dddot{y}}{\frac{\pi}{5}}$ & 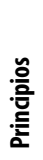 & $\frac{\check{\pi}}{\stackrel{0}{0}}$ & $\begin{array}{l}\text { 옹 } \\
\text { 응 } \\
\text { 옳 }\end{array}$ & 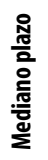 & 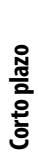 \\
\hline $\begin{array}{l}\text { ¿Conoce usted en qué consiste } \\
\text { el mito de gobierno? }\end{array}$ & 50 & 50 & & & & & & & & & & & & & \\
\hline $\begin{array}{l}\text { ¿Conoce los componentes del } \\
\text { mito de gobierno? }\end{array}$ & & & 23 & 54 & 23 & & & & & & & & & & \\
\hline El mito de gobierno define & & & & & & 11 & 4 & 18 & 18 & 8 & 8 & 33 & & & \\
\hline $\begin{array}{l}\text { En su institución, ¿comunican sus acciones } \\
\text { sobre la base del proyecto general de gobierno? }\end{array}$ & 75 & 25 & & & & & & & & & & & & & \\
\hline $\begin{array}{l}\text { Su plan de comunicación } \\
\text { garantiza las acciones de }\end{array}$ & & & & & & & & & & & & & 43 & 33 & 24 \\
\hline
\end{tabular}

Elaboración: Julio Bravo Mancero. Los valores que se expresan en la tabla son porcentuales.

\section{Conclusiones}

Estudiar la comunicación gubernamental requiere fundamentarla teóricamente para explicar los alcances y la proyección desde la diversidad de enfoques, que van desde el análisis de contenido hasta el análisis del discurso; desde la psicología de la persuasión hasta las teorías de la argumentación. No se pueden enumerar todos los abordajes para ejercer la interpretación de los mensajes emitidos desde la gestión comunicacional. Su definición apunta a lo que se hace desde el territorio y no debe perderse de vista para comprender la misión que implícitamente tiene.

El período de estudio referencia dos momentos (expuestos en la metodología) y no puede excluirse, porque sería un error grave a un tercero que es el teórico, porque la contribución científica fundamentó la investigación y aportó en la generación del debate sobre la temática expuesta. En el camino hubo que afrontar dificultades epistemológicas con relación al objeto de estudio para diferenciarlo y evitar confundir comuni- 
cación gubernamental con relaciones públicas o comunicación corporativa. De allí que los aportes teóricos de los autores tienen asidero y ayudaron en la contextualización.

La sociedad de la información muestra un tipo de ciudadanía con mayor participación e interacción, en la acción y en la toma de decisiones, aspecto que no es ajeno a esta provincia ecuatoriana. La vorágine de la información replanteó formas de acceso ciudadano como garantía de participación y generó inversión para la construcción de plataformas tecnológicas que posibiliten esa premisa.

Lo evidenciado en la investigación indica que en la actualidad la comunicación gubernamental es el eje que conlleva las actividades de gestión, toma de decisiones y de acción, es el nexo entre la búsqueda de participación de la ciudadanía y la necesaria especialización para la oportuna gestión que siempre redundará en la consecución de los objetivos estratégicos. Con precisión Tello (2007) subraya como procesos inevitables para hallar consensos y transformación social. Este documento corrobora la apreciación de una permanente aplicación de estrategias y tácticas de acción política y discursiva para vincular la organización con los usuarios, para dirigir y no persuadir, objetivo inverso utilizado hasta hace poco.

Los resultados de la investigación aclaran el debate de si las instituciones de Chimborazo con la utilización de herramientas de comunicación gubernamental vinculan, comparten, socializan, informan, comunican, participan y orientan la gestión. También arrojó información importante que identificó la formación y los perfiles requeridos para las direcciones de comunicación, el enfoque que la gestión ha tomado, la definición de los especialistas sobre las actividades de comunicación gubernamental, la planificación como piedra angular, el criterio de la importancia del mito de gobierno como un eje sistémico y no en exclusiva como misión-visión.

Los autores investigados le proporcionaron a este documento elementos conceptuales y de operación para comprender lo que significa este capítulo de la comunicación y la forma en que se desarrolla, el espacio que tiene, quiénes están inmersos y cuál es el hilo conductor entre teoría y praxis para su desempeño. Es necesario precisar que Ecuador carece de autores contemporáneos que aporten conocimiento enmarcado en la contemporaneidad para abordar este y otros temas de investigación que orienten el trabajo en territorio, cerca de lo complejo y lejos de la fragmentación.

Este trabajo identifica que pese al avance de la última década el camino recién ha empezado y es necesario mirar la nueva teoría estratégica planeada por Pérez (2001) como un conjunto de directrices que oriente de mejor manera la comunicación gubernamental. Está claro que el nuevo paradigma integrador evita la fragmentación y asigna nuevos roles, los que han pasado desde lo individual hasta lo colectivo. 
La discusión persiste sobre si la acción de la organización y de su líder ${ }^{12}$ va más allá de la interacción con la gente y de la vocación de servicio, o se emplea para que al final de la gestión haya réditos electorales y permanecer en la función o mirar a otra con mayor jerarquía ${ }^{13}$. La respuesta no es ingenua. Todos buscan la continuidad, pero ella dependerá de la gestión-acción, de la comunicación-inclusión, de la utilización de estrategias que generen consensos y no conflictos, y de privilegiar los aspectos plurales y no los singulares.

Los elementos de los resultados, la discusión y la teoría llevan a la comprensión de la comunicación gubernamental como un eje que está creciendo pero que invita a la profundización de su debate, a una especialización a través de programas de posgrado, del plano de la práctica hacia el académico para su fortalecimiento.

\section{Referencias}

Barroso Gómez, José (2013). De la disyuntiva a la articulación: análisis de algunas apuestas científico-prácticas de la comunicación ante la complejidad. Razón y palabra, 17 (2), p. 65-64. Disponible en: http://www.razonypalabra.org.mx/N/N83/ M83/04_Barroso_M83.pdf (consultado: 21 abril 2016).

Bartoli, Annie (1992). Comunicación y organización: la organización comunicante y la comunicación organizada. Barcelona: Paidós Ibérica.

Bel Mallén, José Ignacio (2005). Comunicar para crear valor: la dirección de comunicación en las organizaciones. 2a. ed. Pamplona: Ediciones Universidad de Navarra (EUNSA).

Berlín, Irving (2007). Comunicación gubernamental en los espacios locales: estudio de caso: el ayuntamiento de Mérida, Yucatán, México, durante el periodo (20012004) [tesis]. Santa Cruz de Tenerife, España: Universidad de la Laguna. Servicio de Publicaciones (Serie Tesis doctorales). Disponible en: ftp://tesis.bbtk.ull.es/ ccssyhum/cs428.pdf (consultado: 21 abril 2016).

Costa, Joan (1999). La comunicación en acción: informe sobre la nueva cultura de la gestión. Barcelona: Paidós Ibérica.

Costa, Joan (2007). El dircom hoy: dirección y gestión de la comunicación en la nueva economía. Barcelona: Costa Punto Com.

Elizalde, Luciano \& Riorda, Mario (2013). Comunicación gubernamental 360. Buenos Aires: La Crujía Ediciones.

Escudero, Edilia (2014). Confluencias, interfaces y trayectos en la gestión comunicativa

\footnotetext{
12 Autoridad que tras un proceso de elección popular para el caso de los gobiernos autónomos descentralizados o para funcionarios de designación, como los gobernadores que representan al Ejecutivo en las provincias.

13 Concejalía a alcaldía, alcaldía a prefectura, prefectura a la Asamblea Nacional, Asamblea Nacional a la Presidencia de la República.
} 
dela organización (p.155-198).En:Véliz Montero, Fernando (coord.).Comunicación organizacional: nuevas preguntas para nuevos desafíos. Quito: Ediciones CIESPAL (Intiyán; 67).

Galindo, Jesús (2011). Comunicación estratégica e ingeniería en comunicación social: apunte analítico crítico sobre el libro Estrategias de comunicación (Rafael Alberto Pérez, Ariel Comunicación, Barcelona, 2001). En: Razón y palabra, 75. Disponible en: http://www.razonypalabra.org.mx/N/N75/monotematico_75/17_Galindo_ M75.pdf (consultado: 21 abril 2016).

Marí Sáez, Víctor Manuel (2010). El enfoque de la comunicación participativa para el desarrollo y su puesta en práctica en los medios comunitarios. Razón y palabra, 71. Disponible en: http://www.razonypalabra.org.mx/N/N71/VARIA/27\%20MARI_ REVISADO.pdf (consultado: 21 abril 2016).

Massoni, Sandra (2009). Comunicación estratégica: somos seres en-red-dándonos. FISEC-Estrategias, 5 (12), p. 3-24. Disponible en: http://cienciared.com.ar/ra/ usr/9/254/fisec_estrategias_n12_m1pp3_24.pdf (consultado: 21 abril 2016).

Paoli, Antonio (1997). Comunicación e información. México, D.F.: Trillas.

Pérez, Rafael Alberto (2001). Estrategias de comunicación. Barcelona: Ariel.

Pérez, Rafael Alberto (2012). El estado del arte en la comunicación estratégica. Mediaciones sociales, 10, p. 121-196.

Rizo García, Marta (2012). Imaginarios sobre la comunicación: algunas certezas y muchas incertidumbres en torno a los estudios de comunicación, hoy. Barcelona: Universitat Autonoma de Barcelona. Instituto de la Comunicación.

Sánchez Ruíz, Enrique (2002). La investigación latinoamericana de la comunicación y su entorno social: notas para una agenda. Diá-logos de la comunicación, 64, p. 24-36. Disponible en: http://dialogosfelafacs.net/wp-content/uploads/2012/01/64revista-dialogos-la-investigacion-latinoamericana-de-la-comunicacion.pdf (consultado: 21 abril 2016).

Sánchez, Javier (2013). Comunicar para gobernar. Elizalde, Luciano \& Riorda, Mario (eds.) (2013). Comunicación gubernamental 360 (p. 47-67). Buenos Aires: La Crujía Ediciones.

Silva Echeto, Víctor \& San Eugenio Vela, Jordi de (2014). La investigación en comunicación ante una encrucijada: de la teoría de los campos a la diseminación y diversidad gnoseológica: estudio inicial comparado entre España, Brasil y Chile. Palabra clave, 17 (3), p. 803-827. Disponible en: http://www.redalyc.org/articulo. oa?id=64931834009 [consultado: 21 abril 2016].

Tello, Edgar (2007). Las tecnologías de la información y comunicaciones (TIC) y la brecha digital: su impacto en la sociedad de México. Revista de universidad 
$y$ sociedad del conocimiento, 4 (2), p. 1-8. Disponible en: http://www.uoc.edu/ rusc/4/2/dt/esp/tello.pdf (consultado: 21 abril 2016).

Torres, Rosa María (2005). Sociedad de la información/sociedad del conocimiento. Barcelona: Universitat de Barcelona. Prometheus 21 Investigación y Docencia. Disponible en: http://www.ub.edu/prometheus21/articulos/obsciberprome/ socinfsoccon.pdf (consultado: 21 abril 2016).

Ulloa Tapia, César (2007). Comunicación, cultura y desarrollo. Quito: Ediciones CIESPAL (Intiyán, 47).

Valle, Mónica (2005). Comunicación organizacional, abordajes y perspectivas. Quito: Ediciones CIESPAL (Encuentros).

Véliz Montero, Fernando (coord.) (2014). Comunicación organizacional: nuevas preguntas para nuevos desafíos. Quito: Ediciones CIESPAL (Intiyán; 67). 